\title{
Implementation and Outcomes of a Molecular Tumor Board at Herbert-Herman Cancer Center, Sparrow Hospital
}

\author{
Harsha Trivedi, Devansh Acharya, Ushasree Chamarthy, Joseph Meunier, \\ Hussein Ali-Ahmad, Muhammad Hamdan, James Herman, Gordan Srkalovic
}

Herbert-Herman Cancer Center, Sparrow Hospital

Correspondence: gordan.srkalovic@sparrow.org Tel.: + 15173649400

Fax.: + 15173643687

Received: 27 December 2018 Accepted: 19 April 2019

Key Words: Molecular Tumor Board Precision Medicine - Targeted Therapy Genomic Profiling.

\begin{abstract}
Objective. This paper describes our experience and outcomes from 54 cases presented to the (Molecular tumor board) MTB. Methods. 54 Cases presented between July 2017 and April 2018 were included in this analysis. These patients had different types of cancers that had either failed standard therapy or were expected to fail and physicians were looking for future options for anticipated progression. Patients who had obvious mutations and were candidates for Targeted Agent and Profiling Utilization Registry or Molecular Analysis for Treatment Choice clinical trials were not included. Oncologists presented the cases virtually and Foundation Medicine scientific and clinical team discussed the molecular pathways to find targeted options or trials. Tumor board attendees included oncologists, nurses, pharmacists, mid-level providers, residents and staff of the Cancer Center. Results. Amongst the 54 cases presented $81 \%$ had one or more potentially actionable alteration. $12(22 \%)$ patients received genomically matched therapy as per MTB recommendations. Additional 13 (24\%) patients have options available when they progress. Out of 12 patients who got treatment six are alive at the time of this analysis. Genomically matched therapy or Clinical Trials option were offered to the $46 \%$ of patients based on the MTB discussion. Conclusion. More widespread use of molecular diagnostics, better physician education and multidisciplinary collaboration between the staff involved in diagnosis and treatment, as well as third party payers are necessary for consensus on treatment and care of oncology patients.
\end{abstract}

\section{Introduction}

Precision medicine provides new options for cancer treatments and has become an integral part of oncology clinical practice. For some cancers, such as non-small cell lung cancer (NSCLC), precision medicine and genomic profiling is routinely used to integrate targeted treatments (1). Clinical trials with enrollment based on precision medicine have shown us the utility of targeted thera- pies to block specific molecular pathways activated in cancer (2-5). This is partly possible due to the availability of tumor genomic sequencing technology. These technologies have become more affordable and prevalent, which has led to increasing incorporation of next generation sequencing (NGS)-based comprehensive genomic profiling (CGP) in routine clinical practice. However, the use of CGP for treatment decision guidance is complex for oncologists as it often requires 
complex interpretation of molecular biology and genomic results. With the increase in number of approved and investigational drugs, as well as the number of clinical trials incorporating the expanding knowledge of precision medicine, there is an increasing gap that needs to be filled.

Bridging this gap is largely possible in the setting of molecular tumor boards (MTBs). Multidisciplinary tumor boards in oncology are widely acceptable practice. MTBs include participants with a diverse spectrum of expertise and can provide guidance to oncologists seeking to implement such genomic-based personalized targeted therapy in practice (6-10). MTB review also serves as an educational tool, allowing for evidencebased interpretation of the genomic alterations found in each report. When supported by expert genomicists, bioinformatics specialists, pathologists and molecular oncologists, such discussions can provide rapid and accurate data analyses, comprehensive clinical assessment, as well as consideration of up-to-date availability of relevant clinical trials. Indeed, such MTBs are being established and successfully implemented for treatment decision support and for the guidance of optimal utilization of CGP in the clinic $(6,7,9,10)$.

This article describes the experience of a multidisciplinary MTB, which reviewed molecular profiling reports (Foundation Medicine, Cambridge, MA) of 54 advanced cancer patients with solid tumors who had exhausted or were likely to exhaust standard of care (SOC) options including available clinical trials at our own institution. All patients discussed at the Sparrow Hospital Herbert-Herman Cancer Center (HHCC) MTB between July 2017 and April 2018 are included in this analysis. The tumor board weighed evidence for actionability of genomic alterations identified by the molecular profiling and discussed possible treatment options.

\section{Methods}

The MTB at our cancer center was launched in July 2017 and met twice a month for 60 minutes each month in 2017 and then switched to once a month in 2018. The MTB comprised of medical and radiation oncologists, nursing, pharmacy and clinical trials staff from Sparrow Hospital, and was done virtually with the Foundation Medicine (FM) team including a genomicist and molecular oncologist. At each session four to five cases were presented and discussed in detail. These cases were referred by the treating oncologists. All information was de-identified in compliance with the Health insurance portability and accountability act (HIPAA). Patients and families were informed about the MTB decision making process when their case was referred for the discussion. The recommendations from the MTB were sent to each physician individually by email and maintained on the shared drive for future reference. This was discussed with the patients/families by the treating oncologist. If there was any change in treatment based on the MTB recommendation the new therapy was started only after the patients were educated by the nurses or pharmacist and patients were consented for the treatment.

The patient's treating physician or the senior oncologist, a clinical trials director, or a designated representative (e.g. physician assistant or Clinical trials specialist) presented the patient's case giving concise medical history including the date of diagnosis, type of tumor, therapies received and the relevant markers. This was followed by discussion from the FM genomics scientist and molecular oncologist of the molecular profiling results and implications for each case. Information discussed included the alterations detected in a given sample, their level of characterization and potential actionability. Targeted or immunotherapies 
therapies matched to each alteration detected and approved in the patient's tumor type or in another tumor type, as well as openly enrolling genomically-matched clinical trials were also discussed. This was solely an advisory discussion. The ultimate decision to choose the therapy was left to the treating physicians.

Patients whose cases were selected for the MTB discussion had a range of different solid tumor types $(n=53)$ or lymphoma $(n=1)$. At the time of the MTB they had either failed standard therapy or were expected to fail and their physicians were looking for future options for anticipated progression. Patients who were obvious candidates for any of the open clinical trials at our site including the Targeted Agent and Profiling Utilization Registry (TAPUR) and the National Cancer Institute's Molecular Analysis for Treatment Choice (NCI-MATCH) Study were not selected for MTB discussion. Similarly, patients with clear matches to Food and Drug Administration (FDA) approved therapies in their tumor type were not selected for MTB discussion. Only the cases where the specific genomic mutation was not a direct match to an approved treatment or available clinical trial were selected for presentation to the MTB. By a direct match we meant if the patient's genomic mutation directly matched with the approved therapy. For example, if it was EGFR positive then treat with EGFR targeted treatment or if it was MSI high we will treat with FDA approved Immunotherapy. If after screening patients were eligible based on the genomic target to the list of available drugs on TAPUR or Match they would be enrolled on one of the clinical trials.

Hybrid capture-based comprehensive genomic profiling (Foundation Medicine, Cambridge, MA) was performed on 56 samples from 55 unique patients for 315 genes on submitted FFPE tissue samples $(\mathrm{n}=50)$, for 405 genes on whole blood $(n=3)$, or for
62 genes on circulating tumor DNA isolated from submitted blood samples $(\mathrm{n}=3)$ as previously described (11-13). Most of the patients were sent for genomics when they progressed. However, if it was not possible to get fresh tissue, archival tissue was used from the initial diagnosis. Genomic alterations including base substitutions, insertions/deletions, copy number changes, and rearrangements were assessed, as well as determination of tumor mutational burden (TMB) and microsatellite instability (MSI) status $(14,15)$.

\section{Results}

\section{Patient Characteristics}

Patients presented from July 2017 to April 2018 were included in this analysis. CGP results for a total of 55 patients were presented for MTB discussions. One patient discussed in the MTB had lymphadenopathy only and did not have cancer so was excluded from analysis. All other patients $(\mathrm{n}=$ 54) were heavily pretreated advanced cancer patients who had exhausted or were likely to exhaust SOC options including available clinical trials at our own institution. Only those patients whose oncologist could not easily identify an appropriate genomicallymatched treatment option from the CGP report and thus required the knowledge of the genomics and bioinformatics team were selected for MTB discussion (Figure 1).

Among the tumor types presented the majority of cases were gynecological malignancies $(28 \%, 15 / 54)$ followed by breast carcinoma $(17 \%, 9 / 54)$, colorectal carcinoma (9\%, 5/54), non-small cell lung carcinoma (9\%, $5 / 54)$, or other tumor types $(37 \%, 20 / 54)$.

Demographics of the patients discussed are represented in Table 1. Median age was 64 years (range $37-82$ ) and 69\% (37/54) were females. Patients discussed at this MTB had an average of 2.4 prior lines of therapy be- 


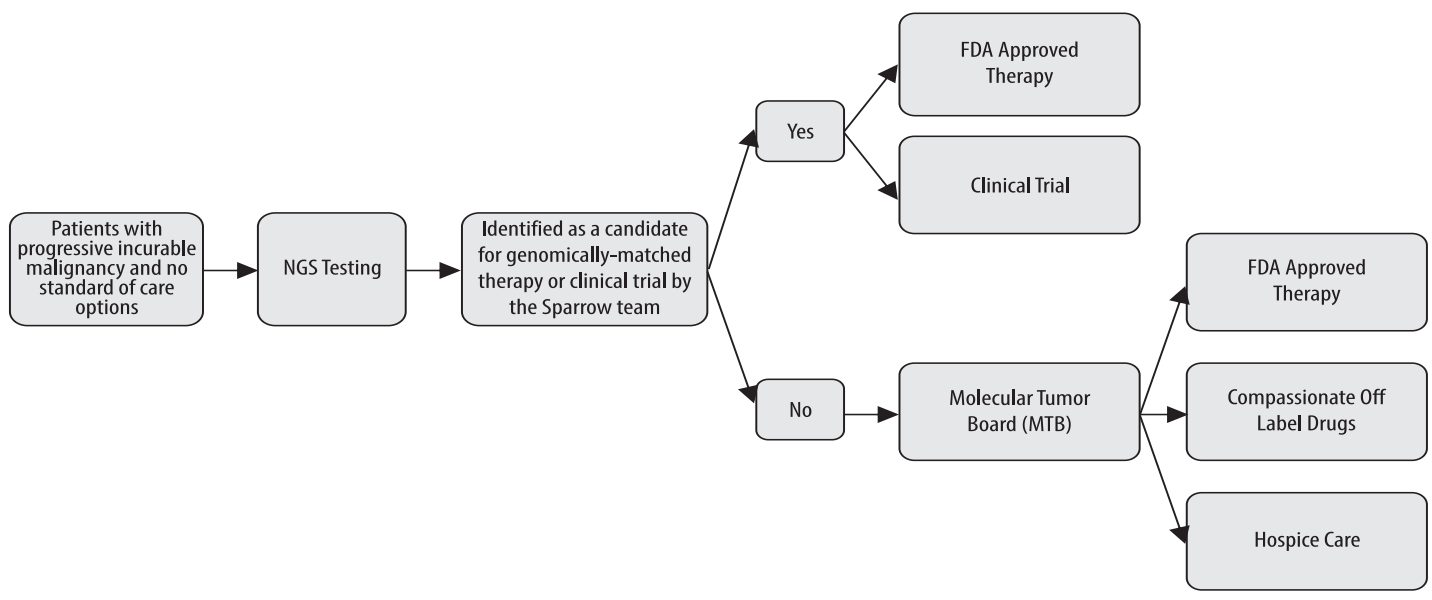

Figure 1. Flow chart depicting selection of patient cases for the Sparrow Health MTB and resulting treatment options.

Table 1. Patient Demographics and Disease History

\begin{tabular}{ll}
\hline Demographics & MTB Patients (\%) \\
\hline No. patients & 54 \\
\hline Median Age (years) & 64 \\
\hline$\geq 65$ years & $26(47)$ \\
\hline$<65$ years & $28(53)$ \\
\hline Gender (Male:Female) & $17(31): 37(69)$ \\
\hline Disease histology & \\
\hline Gynecological & $15(28)$ \\
\hline Breast & $9(17)$ \\
\hline NSCLC & $5(9)$ \\
\hline CRC & $5(9)$ \\
\hline Other & $20(38)$ \\
\hline Number of prior lines of therapy & \\
\hline Mean & 2.39 \\
\hline Median & 2 \\
\hline 1 line & $14(26)$ \\
\hline 2 lines & $18(33)$ \\
\hline 3 lines & $14(26)$ \\
\hline 4 lines & $5(9)$ \\
\hline 5 lines & $1(2)$ \\
\hline 6 lines & $2(4)$ \\
\hline
\end{tabular}

MTB=Molecular tumor board; NSCLC=Non-small cell lung cancer; $\mathrm{CRC}=$ Colorectal cancer. fore CGP was performed; 74\% (40/54) of patients had received $\geq 1$ line of therapy and $15 \%(8 / 54)$ of patients received $\geq 3$ lines of prior therapies. At the time of analysis 32 patients (59\%) were still alive.

\section{Genomic Alterations and Potential Treatment Options Identified}

Of the 54 total patients, $100 \%$ had potentially actionable alteration(s) identified by CGP. An actionable alteration is defined by being linked as either a positive or negative biomarker for an approved therapy or enrollment criteria for an open clinical trial. (Personal communication) Thirteen patients (24\%) had alterations with matched therapy in their tumor type, 25 patients (46\%) had alterations with matched therapy in another tumor type, and 16 patients (30\%) were identified with alterations with a genomically matched clinical trial options (Figure 2). In $76 \%(41 / 54)$ of cases, more than one potentially actionable alteration was identified. 


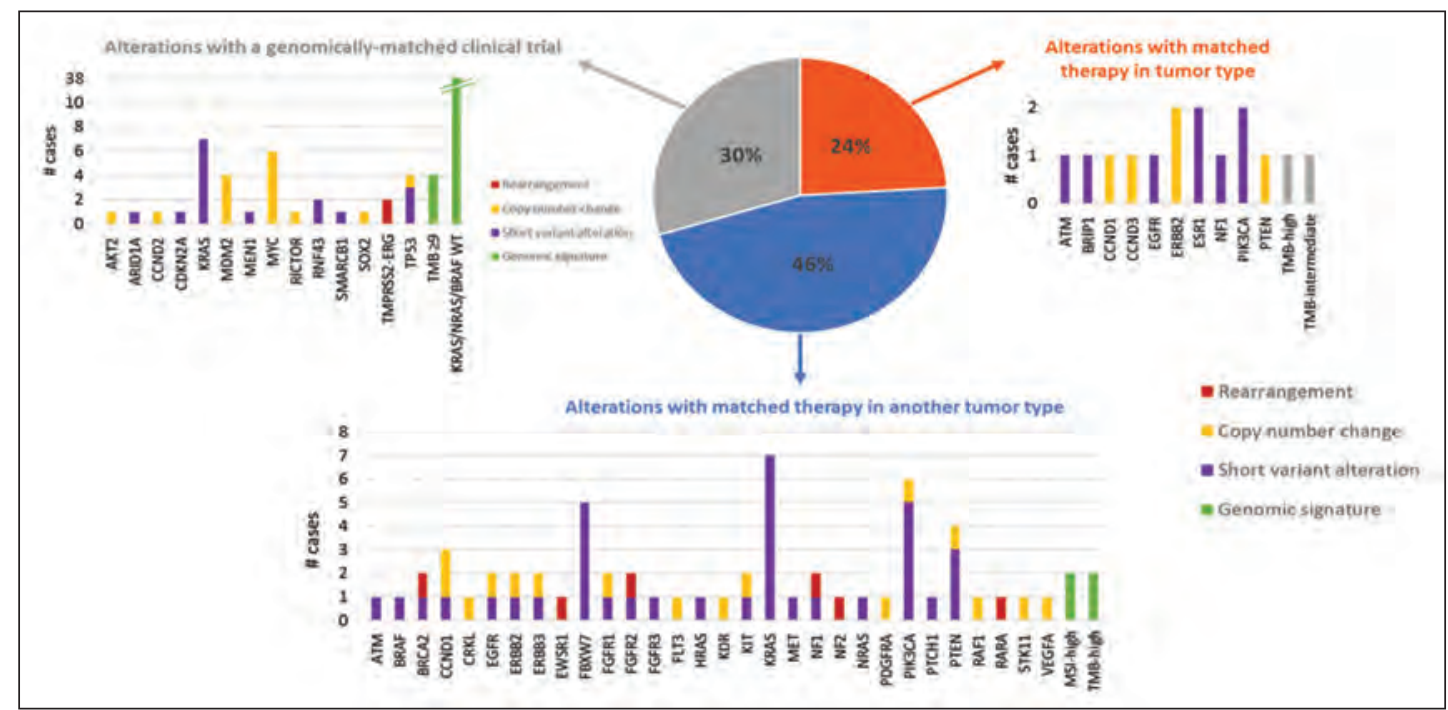

Figure 2. Distribution of potentially actionable alterations identified using CGP. Actionability was assessed at the time of reporting for a given case. Note: a therapy may be approved in a patient's tumor type, but the patient still may not qualify for the therapy based on the specific FDA label. TMB $\geq 9$ mutations/Mb is used as a designator for clinical trial eligibility specifically due to the enrollment criteria for the TAPUR trial pembrolizumab arm, which was open at the time of this study. TMB-intermediate and TMD-high designations for therapy associations were made by Foundation Medicine.

The distribution of potentially actionable alterations identified is shown in Figure 2.

\section{Treatment Assignments and Patient \\ Outcomes}

We further analyzed how MTB discussions influenced the implementation of treatments in our patients. Twelve (22\%) patients received a genomically-matched therapy based on CGP results and MTB discussion (Table 2). Of note, 2 patients received what was assessed to be a genomically-matched treatment based on MTB discussion and the treating physician's discretion, but the therapy received was not listed on the CGP report (Table 2, patients 8 and 11). Out of these 12 patients, 9 had stable disease (SD) as their best response to matched therapy, and 3 had progressive disease (PD) as assessed by recist 1.1 criteria. At the time of follow-up 9 had progressed and 3 maintained SD. The median follow-up period was 17 months. Patients who eventually progressed stayed on treatments between 3 and
15 months. Average time to progression was 7.6 months. Six out of 8 patients who had progressed were alive at the time of analysis (median time to follow up=17 months). An additional 13/54 (24\%) patients are anticipated to receive matched treatment options when they progress on current SOC therapy.

Five out of 54 (9\%) patients had at least one potential genomically-matched therapy option identified, but we could not get approval from insurance $(n=4)$ or the patient did not qualify for available trial(s) primarily due to poor performance status $(n=1)$. Three patients received treatment on label as recommended by MTB. The treating physician did not recognize the direct match and referred to the MTB and the tumor board discussed and recognized the match to the therapies. If those patients were not presented at MTB they would not have gotten these therapies. Six patients (11\%) had genomically-matched options available, but the treating physician chose a different option. This was due to other available agents judged to be more effective than targeted 
Acta Medica Academica 2019;48(1):105-115

Table 2. Clinical and Genomic Characteristics of Patients'Treatment Based on MTB Discussion

\begin{tabular}{|c|c|c|c|c|c|}
\hline Patient & Diagnosis & Matched alteration & Matched targeted therapy & PFS (months) & Best Response \\
\hline 1 & Anus SCC & $\begin{array}{l}\text { KRAS/NRAS/ } \\
\text { BRAF WT }\end{array}$ & Cetuximab on trial & 15 & PD \\
\hline 2 & $\begin{array}{l}\text { Uterus Endometrial } \\
\text { Adeno }\end{array}$ & ERBB2 amplification & Trastuzumab on clinical trial & 6 & SD \\
\hline 3 & $\begin{array}{l}\text { Ovary granulosa } \\
\text { cell tumor }\end{array}$ & $\begin{array}{l}\text { CDKN2A p16INK4a } \\
\text { A60fs* } 89\end{array}$ & Palbociclib on trial & 3 & PD \\
\hline 4 & $\begin{array}{l}\text { Ovary serous } \\
\text { carcinoma }\end{array}$ & ATM D2721M & $\begin{array}{l}\text { Olaparib (FDA-approved } \\
\text { on-label) }\end{array}$ & SD & SD \\
\hline 5 & $\begin{array}{l}\text { Breast carcinoma } \\
\text { (NOS) }\end{array}$ & BRCA2 V1988I & $\begin{array}{l}\text { Olaparib (FDA-approved } \\
\text { off-label) }\end{array}$ & 8 & SD \\
\hline 6 & $\begin{array}{l}\text { Lung } \\
\text { adenocarcinoma }\end{array}$ & $\begin{array}{l}\text { EGFR exon } 19 \text { del + } \\
\text { T790M }\end{array}$ & $\begin{array}{l}\text { Osimertinib (FDA-approved } \\
\text { on-label) }\end{array}$ & SD & SD \\
\hline 7 & Lung SCC & KRAS/NRAS/BRAF WT & Cetuximab on trial & 4 & PD \\
\hline 8 & $\begin{array}{l}\text { Adrenal gland } \\
\text { cortical }\end{array}$ & FGFR2-CIT fusion & Sunitinib on trial" & 5 & SD \\
\hline 9 & Breast ILC & ESR1 Y537N & Fulvestrant on label & 10 & SD \\
\hline 10 & $\begin{array}{l}\text { Ovary serous } \\
\text { carcinoma }\end{array}$ & $\begin{array}{l}\text { MSI-H and TMB } 19 \\
\text { mutations/Mb }\end{array}$ & Pembrolizumab on trial & 12 & SD \\
\hline 11 & $\begin{array}{l}\text { Colon } \\
\text { Adenocarcinoma }\end{array}$ & TMB 8 mutations/Mb & Pembrolizumab off label $^{+}$ & SD & SD \\
\hline 12 & $\begin{array}{l}\text { Breast carcinoma } \\
\text { (NOS) }\end{array}$ & CCND1 amplification & Palbociclib on label & 5 & SD \\
\hline
\end{tabular}

$\mathrm{MTB}=$ Molecular tumor board; $\mathrm{PFS}=$ Progression free survival; $\mathrm{SCC}=$ Squamous cell carcinoma; $\mathrm{WT}=$ Wild Type; $\mathrm{PD}=\mathrm{Prog}$ ressive disease; $\mathrm{SD}=\mathrm{Stable}$ disease; NOS=Not otherwise specified; ILC=Invasive lobular carcinoma; MSI-H=Microsatellite instability high; TMB=Tumor mutational burden; $\mathrm{Mb}=$ Megabase. "This patient was approved for the sunitinib arm of the TAPUR trial based on the FGFR2-CIT fusion alteration detected; however, sunitinib was not one of the matched therapies listed on the CGP report. 'This patient was approved for insurance coverage of off label pembrolizumab based on MTB discussion and TMB of 8 mutations/Mb; however, pembrolizumab was not listed as one of the matched therapies on the CGP report.

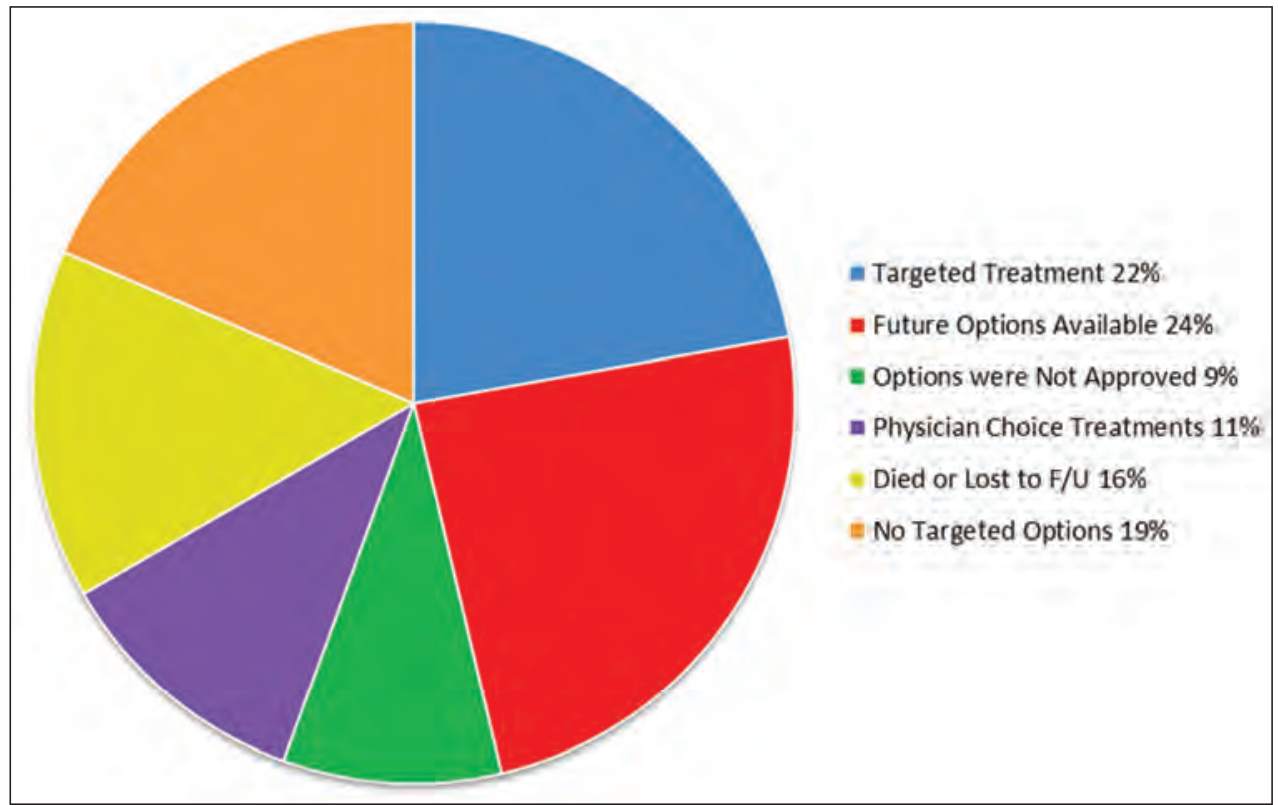

Figure 3. Patient Treatment Assignments Based on Molecular Tumor Board Discussions. 
therapy or inability of the patient to travel to far away sites for treatments. Eight patients (16\%) either died prior to planned treatment or refused further treatments. One patient was lost to follow up and ten patients (19\%) did not have targetable options, even though they had mutations (Figure 3). In total, a genomically matched therapy or clinical trial option was able to be offered to the patient in $81 \%(44 / 54)$ of cases based on the MTB discussion.

\section{Discussion}

There is an increasing body of evidence based on prospective and retrospective studies, case reports, and clinical practice showing that matching targeted agents with genomic alterations improves patient outcomes (16). Clinical reports suggest that $30 \%-80 \%$ of advanced solid tumors harbor potentially actionable genomic variants (17). Meta-analysis of 570 Phase II studies of new anticancer agents, done on 32,149 patients showed that personalized approach correlated with statistically significant higher median responses rates, prolonged median progression free survival and improved overall survival (18). Additional Meta analyses by Schwaederle M et al. also demonstrated benefit for patients treated with personalized matched therapy $(19,20)$. Prospective molecular profiling studies by Stockley et al. demonstrated that treatment with genotype matching in early phase was associated with an increased objective tumor response (13). Wheeler et al. reported that use of CGP to assign therapies in patients with multiple genomic aberrations was associated with longer time to treatment failure and stable disease in patients with refractory malignancies (21). In the multicenter open label phase 2 trial (SHIVA) authors showed that molecularly targeted agents did not improve significantly medium progression free survival (PFS) when compared to physicians' choice of treatment. However, there was a signal for very slight improvement in the PFS, 2.3 versus 2 month in experimental group vs. the control group. This French trial limited molecular alterations to ones identified within 3 molecular pathways (hormone receptor, $\mathrm{PI} 3 \mathrm{~K} / \mathrm{AKT} / \mathrm{mTOR}, \mathrm{RAF} / \mathrm{MEK}$ ) which is the limitation of this study (22).

Another prospective trial, emulating clinical benefits of high throughput genomic analysis in clinical practice, MOSCATO 01 showed that high throughput genomics could improve outcomes in a subset of patients with hard-to-treat cancers. Although only $7 \%$ of successfully screened patients benefited from this approach, we think that with the further refinement of this approach higher or larger number of patients will benefit (23).

However, implementation of genomicbased precision medicine in oncology represents major challenge due to depth of knowledge and expertise required to make decisions which will benefit patients. Obstacles to implementation of precision medicine in clinical practice are particularly high in community practices. They include timeconsuming analyses of results of molecular testing, determining clinical trial eligibility, molecular test selection, determining the optimal time for molecular testing, financial concerns, genetic counseling and particularly patient attitudes. MTBs overcome some of those obstacles by providing necessary expertise in a multidisciplinary setting. The MTB at HHCC was established in July 2017 as cooperative multidisciplinary board in association with FM and in a short period showed to be of great benefit for our patients. We found targetable non-KRAS alterations in $81 \%$ of cases. This is similar to results reported by other molecular tumor boards (39-86\%) (10, 24, 25). These percentages depend upon definition of actionable alterations, and are sensitive to selection bias, since it is expected that physicians will most often 
submit cases for discussion at MTBs they believe have potentially actionable alterations detected by CGP. With advancement in standardization of variant calling and reporting we can expect that differences and biases will be reduced and results from different studies will become more comparable. In the case of our MTB, patients were selected when they did not have a clear choice for a genomicallymatched clinical trial open at HHCC and did not have a direct match to an approved targeted therapy. However, we also discussed and recommended future treatments for patients who were still stable or responding to present treatment. This may be specific for our MTB and could skew results toward higher numbers.

Patients treated based on recommendations from our MTB $(n=12)$ benefited from treatment and those who ultimately progressed $(n=9)$ stayed on treatments between 3 and 15 months (mean 7.6 months). Mean progression free survival on the prior therapy for these patients $(n=9)$ was 4 months. Six out of 8 patients who eventually progressed were still alive at the time of analysis. An additional 3 patients are still being treated with matched therapy and have Stable disease (SD). All these patients had very advanced disease and the only other option was symptom control and Hospice. Data from 126,620 patients extracted from the electronic medical records of 10 hospices in the CHOICE network (Coalition of Hospices Organized to Investigate Comparative Effectiveness) showed that $93.6 \%$ of those patients died within 6 months (26).

One of the characteristics of our MTB was that $13 / 54$ patients were still responding or were stable on previous treatment at the time of the MTB. NGS testing in these patients was done mostly due to patients', families' and physician's anxiety and need to have other available options. Similar observations were made by Schwaederlea et al. and could be considered as a limitation related to the current use of molecular diagnostics. The authors believe that early, and maybe premature testing is related to the time to obtain results (in their case median of 27 days). Consequently, physicians are ordering tests before patients have failed previous treatment (8). In the case of our patients all 13 have potential molecular targets identified by CGP when they progress. We expect that with better and more efficient work flow between local pathology and molecular diagnostic companies' time to obtain results will be significantly reduced and delays will be eliminated. That will taper patients' and physicians' anxiety and bring more appropriate timing of testing.

One of the main concerns from analysis of our MTB results was that 6/54 patients had available molecular targets, but were still treated with chemotherapy by their treating physicians. In addition, 8/54 patients refused recommended molecular treatment or died before it could be applied. Patient's refusal at least partially can be explained by physician's hesitance to use molecular targeted therapy. This is not unexpected since most of the presently practicing oncologists are trained in the era of the "evidence-based medicine" and use of cytotoxic chemotherapy. Although far more informative and accurate than its predecessors of intuition and the "art of medicine", the unfortunate consequence of the approach of "evidence-based medicine" is that outliers are not represented, and they may be unlikely to respond similarly to the average patient for any given treatment. Precision or personalized medicine, in contrast, focuses on the individuals and seeks to improve health outcomes by integrating a huge variety and number of data points, from genomics to environmental and lifestyle factors, in order to provide an individualized approach to health care (27). Although molecular diagnostics use and practice at HHCC is considered advanced, it is still necessary to improve education and 
participation of all treating physicians. MTBs by their structure represent ideal vessels for education, collegiate interaction, multidisciplinary discussion and finally creation of consensus on treatment and care of patients. However, they require full participation of and interaction between all involved participants. Otherwise, opportunities will be missed. There are definite obstacles that need to be overcome, in particular limited available time, especially in busy practices where physicians' income is based on number of patients seen. In order to resolve this important issue, it is necessary to have better understanding of precision medicine by policy makers, third-party payers, hospital administrations, patients and the general public. Development of clinical decision algorithms based on molecular testing and available targeted therapies will make resolution easier. Expected results from precision medicine trials including the National Cancer Institute NCI-MATCH and IMPACT (1-3), and ASCO-TAPUR (17) could help to clarify the role of precision medicine and consequently MTBs in the every day s care of oncology patients.

Need for education and collaboration between providers and third-party payers is emphasized by the number of patients who had molecular targeted options identified, but were refused treatment coverage by payers $(n=4)$, as well as patients who refused treatment $(8 / 54)$. The main reason for these decisions are, in our opinion, costs of the medications, out-of-pocket costs for patients and/or overall costs for third party payers. Bryce et al. (28) had similar experience with their patients at a Mayo MTB where $6 \%$ of the patients with targetable mutations were not able to receive targeted therapy due to insurance denying payments. Hopefully, the increasing trend to incorporate molecular testing and targeted therapy into National Comprehensive Cancer Network (NCCN) and American Society of Clinical Oncology
(ASCO) guidelines will facilitate approval in these cases. It is also our recent experience that some, but not all, third party payers are more inclined to approve targeted therapy based on valid molecular testing.

In 10/54 cases patients did not have targetable options as assessed by the MTB. In these cases therapy or trials were identified linked to a KRAS mutation only (low level of evidence for efficacy) and none of these patients received genomically matched therapy. In 1 additional case the only "genomic match" for actionability was not a directly targetable alteration, but rather option for the KRAS/NRAS/BRAF wild type (WT) cetuximab TAPUR arm. These data argue that CGP identifies potentially actionable alterations in a large majority of patients, but more published evidence for genomically-matched targeted therapy, better access to drugs and trials, more investment into education, better collaboration between all parties vested into patients' care and possibly more appropriate timing of NGS testing (so patients do not die before getting treatment) is needed.

\section{Conclusion}

The MTB is multidisciplinary platform for discussion, treatment recommendations and knowledge acquisition related to genomic testing and precision oncology. Although precision medicine is progressing in breathtaking pace, practice of MTB's is lagging behind. In most of the cases it is limited to large Academic centers. This paper presents model of collaboration between community cancer center and sophisticated technology company that ultimately improves oncology patients' care. This model can be used, with local modifications, in other community centers and bring advantages of precision medicine to more than $80 \%$ of all oncology patients, who are treated in their local communities. 
Acknowledgement: We acknowledge Foundation Medicine team for their help throughout the establishment, operation and scientific discussions of Molecular Tumor board case. Their help and feedback in the preparation of this manuscript is appreciated. We acknowledge the help of Kathryn Edwards, Clinical Research Assistant, Clinical Trials Office, Sparrow Herbert-Herman Cancer Center for the preparation of the manuscript.

Authors' Contributions: Conception and design: GS and HT; Acquisition, analysis and interpretation of data: GS, HT, DA, UC, JM, HA-A, MH and JH; Drafting the article: GS and HT; Revising it critically for important intellectual content: GS and HT; Approved final version of the manuscript: GS, HT, DA, UC, JM, $\mathrm{HA}-\mathrm{A}, \mathrm{MH}$ and $\mathrm{JH}$.

Conflict of Interest: Dr. Srkalovic is on the speakers bureau of Foundation Medicine. None of the other authors have declared any conflict of interest associated with this work.

\section{References}

1. Ersek JL, Black LJ, Thompson MA, Kim ES. Implementing Precision Medicine Programs and Clinical Trials in the Community-Based Oncology Practice: Barriers and Best Practices. Am Soc Clin Oncol Educ Book. 2018(38):188-96.

2. Kim ES, Herbst RS, Wistuba, II, Lee JJ, Blumenschein GR, Jr., Tsao A, et al. The BATTLE trial: personalizing therapy for lung cancer. Cancer Discov. 2011;1(1):44-53.

3. Coyne GO, Takebe N, Chen AP. Defining precision: The precision medicine initiative trials NCIMPACT and NCI-MATCH. Curr Probl Cancer. 2017;41(3):182-93.

4. ASCO. TAPUR: ASCO's First Clinical Trial Addresses Critical Gaps in Understanding of and Access to Targeted Therapies 2015. [cited 2018 Nov 12]. Available from: https://connection.asco.org/ magazine/features/tapur-asco\%E2\%80\%99s-firstclinical-trial-addresses-critical-gaps-understanding-and-access.

5. Kim ES. The Future of Molecular Medicine: Biomarkers, BATTLEs, and Big Data. Am Soc Clin Oncol Educ Book. 2015:22-7.

6. Patel NM, Michelini VV, Snell JM, Balu S, Hoyle AP, Parker JS, et al. Enhancing Next-Generation Sequencing-Guided Cancer Care Through Cognitive Computing. Oncologist. 2018;23(2):179-85.

7. Knepper TC, Bell GC, Hicks JK, Padron E, Teer JK, Vo TT, et al. Key Lessons Learned from Mof- fitt's Molecular Tumor Board: The Clinical Genomics Action Committee Experience. Oncologist. 2017;22(2):144-51.

8. Schwaederle M, Parker BA, Schwab RB, Fanta PT, Boles SG, Daniels GA, et al. Molecular tumor board: the University of California-San Diego Moores Cancer Center experience. Oncologist. 2014;19(6):631-6.

9. Rodriguez-Rodriguez L, Krasna M, Hirshfield KM, Rojas V, Riedlinger G, Ali SM, et al. Feasibility of collaborative precision medicine oncology between academic- and community-based hospitals. Journal of Clinical Oncology. 2017;35(15 suppl):e18015-e. doi: 10.1200/JCO.2017.35.15_ suppl.e18015.

10. Tafe LJ, Gorlov IP, de Abreu FB, Lefferts JA, Liu $\mathrm{X}$, Pettus JR, et al. Implementation of a Molecular Tumor Board: The Impact on Treatment Decisions for 35 Patients Evaluated at Dartmouth-Hitchcock Medical Center. Oncologist. 2015;20(9):1011-8.

11. Frampton GM, Fichtenholtz A, Otto GA, Wang K, Downing SR, He J, et al. Development and validation of a clinical cancer genomic profiling test based on massively parallel DNA sequencing. Nat Biotechnol. 2013;31(11):1023-31.

12. Clark TA, Chung JH, Kennedy M, Hughes JD, Chennagiri N, Lieber DS, et al. Analytical Validation of a Hybrid Capture-Based Next-Generation Sequencing Clinical Assay for Genomic Profiling of Cell-Free Circulating Tumor DNA. J Mol Diagn. 2018;20(5):686-702.

13. Stockley TL, Oza AM, Berman HK, Leighl NB, Knox JJ, Shepherd FA, et al. Molecular profiling of advanced solid tumors and patient outcomes with genotype-matched clinical trials: the Princess Margaret IMPACT/COMPACT trial. Genome Med. 2016;8(1):109.

14. Chalmers ZR, Connelly CF, Fabrizio D, Gay L, Ali SM, Ennis R, et al. Analysis of 100,000 human cancer genomes reveals the landscape of tumor mutational burden. Genome Med. 2017;9(1):34.

15. Fabrizio DA, George TJ, Jr., Dunne RF, Frampton G, Sun J, Gowen K, et al. Beyond microsatellite testing: assessment of tumor mutational burden identifies subsets of colorectal cancer who may respond to immune checkpoint inhibition. J Gastrointest Oncol. 2018;9(4):610-7.

16. Moscow JA, Fojo T, Schilsky RL. The evidence framework for precision cancer medicine. Nat Rev Clin Oncol. 2018;15(3):183-92.

17. Mangat PK, Halabi S, Bruinooge SS, GarrettMayer E, Alva A, Janeway KA, et al. Rationale and Design of the Targeted Agent and Profiling Uti- 
lization Registry Study. JCO Precision Oncology. 2018(2):1-14.

18. Schwaederle M, Zhao M, Lee JJ, Eggermont AM, Schilsky RL, Mendelsohn J, et al. Impact of Precision Medicine in Diverse Cancers: A MetaAnalysis of Phase II Clinical Trials. J Clin Oncol. 2015;33(32):3817-25.

19. Schwaederle M, Zhao M, Lee JJ, Lazar V, Leyland-Jones B, Schilsky RL, et al. Association of Biomarker-Based Treatment Strategies With Response Rates and Progression-Free Survival in Refractory Malignant Neoplasms: A Meta-analysis. JAMA Oncol. 2016;2(11):1452-9.

20. Jardim DL, Schwaederle M, Wei C, Lee JJ, Hong DS, Eggermont AM, et al. Impact of a BiomarkerBased Strategy on Oncology Drug Development: A Meta-analysis of Clinical Trials Leading to FDA Approval. J Natl Cancer Inst. 2015;107(11).

21. Wheler JJ, Janku F, Naing A, Li Y, Stephen B, Zinner R, et al. Cancer Therapy Directed by Comprehensive Genomic Profiling: A Single Center Study. Cancer Res. 2016;76(13):3690-701.

22. Le Tourneau C, Delord JP, Goncalves A, Gavoille C, Dubot C, Isambert N, et al. Molecularly targeted therapy based on tumour molecular profiling versus conventional therapy for advanced cancer (SHIVA): a multicentre, open-label, proof-of-concept, randomised, controlled phase 2 trial. Lancet Oncol. 2015;16(13):1324-34.
23. Massard C, Michiels S, Ferte C, Le Deley MC, Lacroix L, Hollebecque A, et al. High-Throughput Genomics and Clinical Outcome in Hard-to-Treat Advanced Cancers: Results of the MOSCATO 01 Trial. Cancer Discov. 2017;7(6):586-95.

24. Burkard ME, Deming DA, Parsons BM, Kenny PA, Schuh MR, Leal T, et al. Implementation and Clinical Utility of an Integrated Academic-Community Regional Molecular Tumor Board. JCO Precision Oncology. 2017;1(1):1-10.

25. Harada S, Arend R, Dai Q, Levesque JA, Winokur TS, Guo R, et al. Implementation and utilization of the molecular tumor board to guide precision medicine. Oncotarget. 2017;8(34):57845-54.

26. Harris PS, Stalam T, Ache KA, Harrold JE, Craig $\mathrm{T}$, Teno J, et al. Can hospices predict which patients will die within six months? J Palliat Med. 2014;17(8):894-8.

27. Trivedi H KH, Treece T, Audeh W, Srkalovic G. Changing the Landscape of Clinical-Genomic Oncology Practice. Acta Med Acad. 2019;48(1):617.

28. Bryce AH, Egan JB, Borad MJ, Stewart AK, Nowakowski GS, Chanan-Khan A, et al. Experience with precision genomics and tumor board, indicates frequent target identification, but barriers to delivery. Oncotarget. 2017;8(16):27145-54 . 